\title{
Migración interna y fecundidad de las mujeres colombianas
}

\section{Internal migration and fertility of colombian women}

DOI: http://dx.doi.org/10.17981/cultedusoc.12.1.2021.06

Recibido: 17 de diciembre de 2019 Aceptado 16 de junio de 2020 Publicado: 1 de diciembre de 2020

\author{
Laura Alejandra Guerrero-Cely \\ Universidad Militar Nueva Granada. Bogotá, D.C. (Colombia) \\ u2101715@unimilitar.edu.co
}

Alisson Zharick Bejarano-Carreño

Universidad Militar Nueva Granada. Bogotá, D.C. (Colombia)

u2101697@unimilitar.edu.co

Adriana Carolina Silvia-Arias

Universidad Militar Nueva Granada. Bogotá, D.C. (Colombia)

adriana.silva@unimilitar.edu.co

Para citar este artículo:

Guerrero-Cely, L., Bejarano-Carreño, A. y Silvia-Arias, A. (2021). Migración interna y fecundidad de las mujeres colombianas. Cultura, Educación y Sociedad, 12(1), 85-104. DOI: http://dx.doi.org/10.17981/cultedusoc.12.1.2021.06

\section{Resumen}

La migración interna femenina en Colombia ha tenido repercusiones en la redistribución demográfica de la población. Esta movilidad ha incidido en las preferencias reproductivas de las mujeres. El artículo tiene como propósito analizar la relación entre la migración interna y la fecundidad de las mujeres colombianas. A partir de la información proporcionada por Profamilia de la Encuesta Nacional de Demografía y Salud (ENDS) del 2015, se caracteriza el perfil de las mujeres migrantes internas colombianas y se elaboran estimaciones fundamentadas en un modelo logístico para prever los factores incidentes en la mayor probabilidad de que una mujer en condición de migrante interna pueda tener hijos. Los resultados muestran que los factores socioeconómicos propios de las migrantes internas están asociados a una mayor probabilidad de tener hijos: mujeres en edades consideradas de mayor fertilidad, menor estrato, que viajaron acompañadas, vivían en pareja, y migraron por razones familiares o por salud. Todos estos factores asociados a la migración femenina afectaron su adaptación en los lugares de destino, así como sus preferencias reproductivas. Las políticas migratorias internas deberían dirigirse a apoyar el proceso de adaptación de las migrantes en los lugares de destino, así como facilitar su retorno a los territorios.

Palabras clave: Demografía; migración interna; fecundidad; condiciones de vida; políticas migratorias

\section{Abstract}

Internal female migration in Colombia has had repercussions on the demographic redistribution of the population. This mobility has affected the reproductive preferences of women. The purpose of the article is to analyze the relationship between internal migration and the fertility of Colombian women. Based on the information provided by Profamilia from the 2015 National Demographic and Health Survey (ENDS), the profile of internal Colombian migrant women is characterized and estimates based on a logistic model are prepared to predict the incident factors in the highest probability that a woman who is an internal migrant can have children. The results show that the socioeconomic factors typical of internal migrants are associated with a greater probability of having children: women of ages considered to be of higher fertility, lower stratum, who traveled with someone, lived with a partner, and migrated for family or health reasons. All these factors associated with female migration affected their adaptation to their destination, as well as their reproductive preferences. Internal migration policies should be aimed at supporting the process of adaptation of migrants in the places of destination, as well as facilitating their return to the territories.

Keywords: Demography; internal migration; fertility; life conditions; migration policies 


\section{INTRODUCCIÓN}

Las migraciones han sido un fenómeno constante a lo largo del tiempo, las cuales se originan en contextos políticos, sociales y económicos (González, 2012). Una de las razones más frecuentes para migrar en los últimos años, ha sido la reunificación familiar, sobre todo de las mujeres, con el propósito de proporcionar una mejor calidad de vida al hogar, y principalmente de los hijos (Willers, 2016). En el análisis de la evolución de los flujos migratorios, se ha identificado que las características, intenciones y necesidades difieren entre los diferentes flujos de migrantes. Lo anterior ha permitido reconocer patrones de migración mundial, así como el modo en que influyen distintos factores y políticas del lugar de origen y destino (Organización de las Naciones Unidas-ONU, 2017). En Latinoamérica los altos niveles de pobreza, desigualdad y escasez de oportunidades dieron origen a un alto flujo de migrantes tanto internos como internacionales. La mayor parte de los flujos migratorios de las últimas décadas se presentaron hacia países desarrollados (Ramírez, Zuluaga y Perilla, 2010).

Para el caso colombiano, la migración está relacionada con las transformaciones de la sociedad, la percepción de seguridad y la búsqueda de mejores ingresos y oportunidades laborales (Asociación Probienestar de la Familia Colombiana-Profamilia, 2016). En el perfil migratorio colombiano, la edad, la coyuntura económica, la consolidación de redes sociales y el nivel educativo de los migrantes, se destacan como los principales determinantes de la transición demográfica (Ramírez et al., 2010). La migración interna en Colombia se ha relacionado principalmente con factores como el conflicto armado, el desempleo y el acceso limitado a servicios sociales como la educación y la salud (Profamilia, 2016). Las migraciones internas en Colombia prevalentemente han sido de contextos rurales a urbanos. Las ciudades, por lo general, no están en capacidad de acoger a todos los migrantes, por lo que las necesidades que motivaron la migración terminan trasladándose a los lugares de destino. En este sentido, la migración incrementa las disparidades territoriales (Corona, 2000).

La movilidad poblacional ha significado una mejora en el bienestar de las personas. Sin embargo, no todos los movimientos migratorios se presentan en contextos favorables. Por tal razón, la migración en condiciones de alta vulnerabilidad social genera dificultades de inserción para los migrantes y reproduce la violencia de los contextos de origen (Organización Internacional para las Migraciones-OIM, 2018). Adicionalmente, el aumento en los flujos migratorios ha ido acompañado de un descenso en la fecundidad mundial desde décadas atrás. La Tasa Global de Fecundidad (TGF) en Colombia fue de 1.93 hijos por mujer durante 2017, una tasa menor a la considerada de reemplazo poblacional de 2.1 hijos por mujer (Cabella \& Nathan, 2018).

Como consecuencia del descenso de la fecundidad, el envejecimiento de la población aumentó la proporción relativa de los adultos mayores, modificando la pirámide poblacional (Fondo de Población de las Naciones Unidas en Venezuela-UNFPA, 2006). De modo que, la fecundidad futura de los territorios, está restringida por las disparidades de la composición demográfica de la población y por la recomposición de los territorios ocasionada por la migración (Cabella \& Nathan, 2018). 
Por tanto, la transición demográfica a menores niveles de fecundidad ha incidido en la conformación familiar, modificando la estructura y núcleos familiares convencionales, tanto en el lugar de origen como en el de llegada. Por lo que es pertinente reconocer el papel de la familia, y esencialmente el papel de la mujer, en la construcción del entorno en el que se desarrollan los individuos (Lagomarsino, 2005). La reproducción del capital social dependerá de las condiciones de vida de los individuos al momento de migrar y de su capacidad de adaptación en el lugar de destino; apoyados no solo de los recursos económicos, sino de las redes y apoyos sociales que disponen en los lugares de destino (Acuña y Silva, 2016). Así pues, para analizar las pautas reproductivas de las mujeres migrantes es preciso considerar aspectos relacionados con el nivel socioeconómico y el contexto social en los que se originan los movimientos migratorios. De igual manera, los flujos migratorios al ser una parte intrínseca del desarrollo humano y las disparidades territoriales, deben estar incluidos dentro de los fenómenos demográficos que se consideran al tomar decisiones de política pública (Perilla, 2011).

En Colombia existe un alto volumen de migración interna y un notable descenso de la fecundidad, especialmente de mujeres migrantes. Durante el 2015, el 33.54\% de las mujeres migrantes internas tuvieron hijos, mientras que esta proporción fue el 37.22\% para las migrantes internacionales y el $23.81 \%$ para las mujeres que no migraron. El propósito de este artículo es analizar los factores asociados a la migración interna femenina y la fecundidad. Los datos provienen de la Encuesta Nacional de Demografía y Salud (ENDS) de 2015, que recolecta datos acerca de la salud sexual y reproductiva de la población colombiana. Las mujeres migraron al interior de Colombia en sus edades más fértiles y por razones familiares, acompañadas, provenientes de hogares de estratos bajos, en pareja, con bajos niveles educativos e ingresos. Estas características ponen de manifiesto los condicionantes sociales en que ocurre durante el periplo migratorio de las mujeres colombianas, así como la escasez de recursos económicos y sociales en los lugares de destino para tener hijos.

El artículo se organiza como se menciona a continuación. En la siguiente sección, se presenta la revisión de la literatura de los principales referentes teóricos y empíricos para analizar la relación entre migración y fecundidad. Luego, se presenta el apartado metodológico y el análisis de los resultados. Posteriormente, se presentan las conclusiones y recomendaciones para la definición de políticas migratorias.

\section{REVISIÓN DE LA LITERATURA}

La literatura ha abordado desde diferentes perspectivas la relación entre la migración y la fecundidad. En esta sección se exponen investigaciones que abordan las causas de la migración, en específico de la migración interna femenina, así como los factores asociados a la fecundidad, para finalmente exponer las teorías que abordan la relación entre migración y fecundidad.

\section{Movimientos migratorios}

Los movimientos migratorios surgen como respuesta a distintas problemáticas económicas y sociales relacionadas con la disparidad entre los niveles de ingresos, el empleo y el 
bienestar social en los distintos territorios, así como con la creciente oferta de mano de obra en economías menos desarrolladas que se encuentran en condiciones de mayor pobreza o en zonas de conflicto armado (Palacios, 2010). Históricamente, han existido movimientos poblacionales a causa de la integración de las comunidades locales y globales. Este flujo de migrantes se convierte en un eje de transformaciones sociales y culturales, tanto en el lugar de origen, como en el lugar de destino del migrante. Los flujos migratorios pueden contribuir con el crecimiento económico y el bienestar social, pero también pueden agudizar el estancamiento y la desigualdad (Abu, 2007). Por lo anterior, los factores asociados a la migración son los que determinan el desarrollo y crecimiento económico de los países, así como se constituyen en uno de los principales desafíos a enfrentar por los gobiernos (Comisión Económica para América Latina y el Caribe-CEPAL, 2006).

Las migraciones internas suelen diferenciarse entre forzadas y voluntarias, a partir de la principal razón que motivó el traslado. Algunas personas se ven forzadas a dejar su lugar de residencia cuando son desplazados por desastres naturales, por violencia, como es el caso de los desplazados por los conflictos armados internos. Por el contrario, los flujos de migrantes voluntarios se originan de la decisión de mejorar las condiciones de vida personales y familiares en los lugares de destino (Olea, 2011). Cabe destacar que existen perfiles migratorios distintivos en los que predomina una población relativamente más joven, dotada de mayor capacidad de iniciativa y con un nivel de formación superior, con respecto a la población del lugar de origen. Por lo tanto, con la migración el territorio pierde capital humano que pasa a contribuir a los mercados laborales en el lugar de destino (Alonso, 2011).

La mayor propensión migratoria de la población joven se aduce por el ingreso a la universidad, la inserción al mercado de laboral, la unión en pareja y la formación de un hogar. No obstante, hay otros factores subjetivos asociados a la noción del ciclo vital, como la menor aversión al riesgo en esta fase de su proyecto de vida (Rodríguez, 2015). Lo anterior está directamente relacionado con la mayor participación de mujeres en los flujos migratorios. La creciente feminización de la migración en los últimos años, ya que conforman alrededor de la mitad de la población migrante, y que ocurre en la edad de mayor fertilidad, por lo que se considera relevante analizar la migración femenina (Vicente, 2006).

En años más recientes, se ha evidenciado una creciente heterogeneidad en el perfil de las mujeres migrantes. El perfil de la migración femenina se encuentra estrechamente relacionado con los cambios en las relaciones familiares y en el matrimonio. En general, las mujeres migran por voluntad propia y sin acompañantes (CEPAL, 2006).

\section{Migración interna}

La migración interna hace referencia al desplazamiento poblacional que tiene lugar al interior de un país. Este proceso, en su dimensión cultural, apoya la construcción del tejido social de manera que se vuelve un instrumento que ayuda a forjar las transformaciones demográficas a lo largo de un territorio. De esta forma, las migraciones internas modifican la estructura y dinámica poblacional, e incluso transforman las tradiciones y la identidad local (Castro, 2016). 
La migración interna en Colombia se ha presentado en gran medida desde áreas rurales, las cuales se han caracterizado por precarias condiciones de calidad de vida y han sido territorios más afectados por el conflicto armado interno. Estas condiciones de desplazamiento forzado por la violencia obstaculizan el desarrollo humano y el bienestar social en el mediano y largo plazo. Además, para los desplazados forzados por la violencia, son reducidas las oportunidades para insertarse laboralmente en los lugares de destino, ya que los oficios que realizaban en las áreas rurales no se requieren para las ocupaciones en las zonas urbanas (Ibáñez y Velásquez, 2008).

Una alta proporción de los migrantes internos en Colombia, prevalentemente mujeres jóvenes, migraron debido al conflicto armado interno. A estas mujeres migrantes se les altera su entorno, los patrones reproductivos, el desempeño laboral, entre otros factores; lo que a su vez causa profundas consecuencias económicas y sociales (Ruiz, 2011). De esta forma, los condicionantes sociales de las mujeres migrantes limitaron la inserción a empleos formales, que fue aún más limitada para las desplazadas forzadas. El nivel educativo alcanzado determina las posibilidades de ocuparse y permanecer empleadas. Las mujeres desplazadas forzadas tuvieron menor nivel educativo que las otras mujeres migrantes, lo cual dificultó más su inserción laboral en los lugares de destino (Silva y Sarmiento, 2013).

El proceso migratorio ha concentrado el capital humano en las ciudades más grandes y prósperas. El acceso efectivo a la educación ha sido limitado en las zonas expulsoras, donde la búsqueda de una mejor educación ha sido un motivo recurrente para migrar (Romero, 2015). De esta forma, aunque es útil para los migrantes y beneficioso para las regiones pujantes, erosiona el capital humano de las regiones más pobres y menos desarrolladas (Rodríguez, 2008). Por tanto, la migración interna aumenta las desigualdades sociales territoriales (Rodríguez, Turra, Bonifácio y Wajnman, 2013). No obstante, los migrantes no son una muestra representativa y homogénea de la población, ya que operan mecanismos de selectividad, que puede ser aún más disímiles entre las mujeres migrantes (Rodríguez, 2004).

\section{Fecundidad}

La fecundidad desempeña un papel importante en la evolución demográfica, puesto que incide en otras dimensiones como la económica, social y cultural, que a su vez determinan el comportamiento individual. Las preferencias de fecundidad son el resultado de valores culturales, estructuras sociales y económicas del núcleo social en el que viven los individuos (Iriso y Reher, 1987). La Tasa Global de Fecundidad (TGF) en Colombia presentó una reducción del 65\% en tan solo 50 años. A lo anterior se asocian factores como el incremento de la participación de la mujer en el mercado laboral, la mayor proporción de parejas en unión consensual y la decisión de reducir el tamaño de los hogares (Silva y González, 2007).

Asimismo, la reducción de la fecundidad se asocia con una mayor oferta de anticonceptivos, a la implementación de programas de planificación familiar y la adopción de nuevos patrones reproductivos como respuesta a un período de continuas crisis económicas (Schkolnik y Chackiel, 2004). Incluso se considera que otros factores externos afectaron 
los niveles de fecundidad, como la violencia, avances en la medicina, entre otros (Mesa y Junca, 2011). En contraposición, entre los factores que aumentan la probabilidad de ser madre se considera la conyugalidad, la edad de la primera relación sexual y la baja tasa de utilización de anticonceptivos. Se demostró que estos tres factores determinan la propensión al embarazo (Rodríguez, 2007).

Además, las altas tasas de fecundidad se han asociado a la población de menores ingresos, bajo nivel de educación y los que se ubicaron en zonas rurales o de la periferia urbana, donde se dificulta el acceso a la información sobre planificación familiar y salud sexual y reproductiva. La población que aportó más a la caída de la fecundidad fue principalmente los que lograron niveles más altos de educación y quienes residieron en las áreas urbanas (Chackiel, 2004).

\section{Relación entre migración y fecundidad}

A continuación, se presentan algunas perspectivas que abordan la relación entre migración y fecundidad. La perspectiva teórica de adaptación de la fecundidad se refiere a que las preferencias reproductivas de las migrantes se adaptan gradualmente al nivel de fecundidad de las mujeres de los lugares de destino. Por tanto, se asume que las migrantes en los lugares de destino tuvieron mayor acceso a planificación familiar, así como mejores condiciones de vida, lo que incide en que los niveles de fecundidad sean semejantes a los de las mujeres nativas (Canales, 1999).

Otro factor que contribuye al incremento de la fecundidad de las migrantes es la reunificación familiar. Eventualmente, se considera la posibilidad de que toda la familia migre tras la estabilización económica en el lugar receptor. Sin embargo, la adaptación e integración de los migrantes trae nuevos desafíos a las sociedades de destino, principalmente para mujeres y niños (Olea, 2011).

En la actualidad, las mujeres migrantes se han caracterizado por ser jóvenes con bajas tasas de fecundidad, que podrían incrementar la fecundidad tras su migración e integración. Esto implica una pérdida de mujeres en edad reproductiva, así como limita el crecimiento poblacional de largo plazo en los lugares de origen. De esta manera, se evidencia la compleja interrelación de los procesos migratorios con las preferencias de fecundidad (Rodríguez et al., 2013).

\section{MetodoloGía}

\section{Datos}

La información proviene de los microdatos de la Encuesta Nacional de Demografía y Salud (ENDS) realizada en 2015 por Profamilia (2016). Esta encuesta ha sido publicada desde 1990, con una periodicidad de cinco años y proporciona datos acerca de la salud sexual y reproductiva de los colombianos (Bejarano, Guerrero y Silva, 2019). Asimismo, aporta información sobre la dinámica demográfica en Colombia, a través de un muestreo probabilístico en 44.614 hogares con representatividad nacional (Ministerio de Salud y Protección Social y Profamilia, 2015). 
En particular, se analizaron los módulos de miembros del hogar y niños; dichos módulos contienen variables relacionadas con el cambio de residencia y las razones de la migración, además de los rasgos que caracterizan las preferencias de fecundidad y el contexto socioeconómico de las migrantes.

\section{Universo de estudio}

El universo de estudio se delimitó a una muestra de 6876 mujeres colombianas migrantes en edad fértil, entre los 13 y los 49 años que fueron entrevistadas en la Encuesta Nacional de Demografía y Salud-ENDS (Profamilia, 2016). Se definió como migrante, una mujer colombiana que vivió en los últimos cinco años (desde 2010) en más de un lugar. La composición de esta muestra de 6876 mujeres colombianas que al menos una vez cambiaron de lugar de residencia en los últimos cinco años (desde 2010) fue: 6481 de ellas se consideraron migrantes internas porque el cambio fue dentro del país, y 395 de ellas se consideraron migrantes internacionales porque vivieron en otro país.

\section{Método}

En el artículo se presenta un análisis descriptivo y la estimación de los factores relacionados con la probabilidad de que una mujer migrante en Colombia tuviera o no un hijo. De esta forma, se realiza un análisis empírico de las mujeres migrantes y la fecundidad en Colombia. Posteriormente, mediante un modelo logístico se estima la probabilidad de que una migrante tenga o no un hijo, ya que este tipo de modelos permite obtener estimaciones de la probabilidad de un suceso, así como los factores que determinan dicha probabilidad (Gujarati y Porter, 2010).

A continuación, se presenta la ecuación (1) del modelo estimado:

$$
P_{i}=E\left(y=\frac{1}{X_{i}}\right)=\frac{1}{1+e^{-\left(\beta_{0}+\beta_{i} X\right)}}
$$

La variable dependiente toma el valor de $1(y=1)$ si la mujer tiene al menos un hijo, y toma el valor de $0(y=0)$ en caso contrario. Así, la probabilidad de tener un hijo se encuentra asociada a un conjunto de variables explicatorias que se resumen en el vector $X$. La principal hipótesis de este artículo es que la condición migratoria y la causa de la migración, afectaron los comportamientos reproductivos de las mujeres migrantes colombianas. Se consideraron como variables explicats: el tipo de migrante (Mig): interna o internacional; el motivo de la migración (Raz): razones familiares, conflicto armado interno, desastres naturales, buscando mejores ingresos, educación, salud, otros; la edad cuadrática (Edad, $E d a d^{2}$ ), ya que existe mayor probabilidad de tener un hijo en la mitad del ciclo de vida, por lo que la relación entre edad y fecundidad no sería lineal; si vivía o no con el cónyuge (EstCivil) y si migró sola o acompañada (Acom), debido a que estos factores afectan las preferencias y la prevalencia de la fecundidad en el lugar de destino; la zona de residencia (Zona): rural o urbana; el estrato de residencia (Estrato): 1 al 6; el nivel educativo (Edu): sin educación, primaria, secundaria, superior; y si percibió algún ingreso: sí/no (Ing). 
Por lo que, de acuerdo con las variables explicativas consideradas, la ecuación (2) de la probabilidad de tener hijos de las migrantes a estimar es:

$$
P_{i}=E\left(y=1 / X_{i}\right)=1 /\left(\begin{array}{c}
1_{1+\beta_{0}+\beta_{1} \text { Mig }+\beta_{2} E d a d+\beta_{3} E d a d^{2}+\beta_{4} E d u+\beta_{5} R a z+\beta_{6} A c o m}^{\left.+\beta_{7} E s t C i v i l+\beta_{8} \text { Zona }+\beta_{9} E s t r a t o+\beta_{10} \text { Ing }\right)}
\end{array}\right)
$$

Además, se calcularon los efectos marginales de cada uno de estos factores en la probabilidad de que tengan hijos las mujeres migrantes colombianas. Con tal propósito, el perfil de la mujer migrante que se tendrá en cuenta como base para el análisis será: una que cambió de residencia al menos una vez entre 2010 y 2015 al interior del país, que tenía la edad promedio de las mujeres en la muestra, que no tenía educación, estrato 1, que la razón por la que migró fue por el conflicto armado interno, viajó acompañada y vivía en pareja en una zona urbana.

\section{Resultados}

Para el análisis estadístico de la fecundidad de las mujeres migrantes colombianas de la muestra, en la Figura 1 se presenta la proporción de mujeres con o sin hijos por estatus migratorio. De esta forma, una de cada cuatro mujeres en edad fértil tuvo hijos (25.28\%), siendo menor la prevalencia de mujeres con hijos para las migrantes en 10 puntos porcentuales (23.81\% de las mujeres no migrantes frente al $33.76 \%$ de las mujeres migrantes). Lo anterior pone de manifiesto las diferencias en la fecundidad entre migrantes y no migrantes.

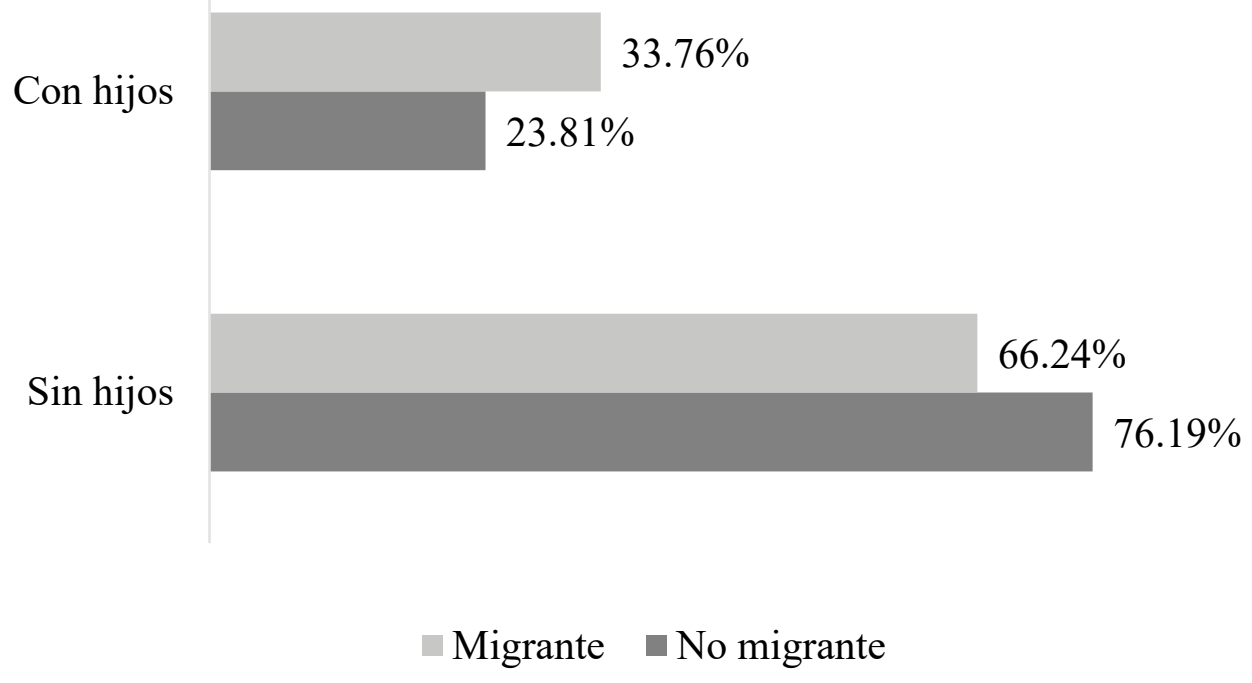

Figura 1. Porcentaje de mujeres colombianas por estatus migratorio y fecundidad. Fuente: ENDS (2015). Cálculos propios. 
A lo anterior, han contribuido factores como el incremento al acceso de métodos anticonceptivos, la mayor vinculación de la mujer al trabajo y un mayor nivel educativo (Silva y González, 2007). No obstante, la llegada de este grupo de migrantes puede contrarrestar en el mediano y largo plazo el deterioro de la pirámide poblacional, que afecta el mercado laboral y el sistema pensional (León, 2005).

TABLA 1.

Perfil de las mujeres colombianas por condición migratoria y fecundidad

\begin{tabular}{|c|c|c|c|}
\hline & Sin hijos & Con hijos & Total \\
\hline \multicolumn{4}{|l|}{ Edad } \\
\hline \multirow[t]{2}{*}{ Edad promedio } & 27.922 & 26.171 & 27.213 \\
\hline & $(9.003)$ & $(5.893)$ & $(8.167)$ \\
\hline \multicolumn{4}{|l|}{ Razón para migrar } \\
\hline Conflicto armado & 0.06 & 0.062 & 0.061 \\
\hline Desastres naturales & 0.004 & 0.002 & 0.003 \\
\hline Buscando mejorar ingresos & 0.306 & 0.307 & 0.306 \\
\hline Educación & 0.129 & 0.035 & 0.097 \\
\hline Salud & 0.021 & 0.035 & 0.026 \\
\hline Razones familiares & 0.456 & 0.538 & 0.484 \\
\hline Otros & 0.023 & 0.022 & 0.023 \\
\hline \multicolumn{4}{|l|}{ Estado civil } \\
\hline Con pareja & 0.443 & 0.758 & 0.549 \\
\hline Sin pareja & 0.557 & 0.242 & 0.451 \\
\hline \multicolumn{4}{|l|}{ ¿Viaja con su pareja? } \\
\hline No & 0.575 & 0.486 & 0.545 \\
\hline $\mathrm{Si}$ & 0.425 & 0.514 & 0.455 \\
\hline \multicolumn{4}{|l|}{ Nivel educativo } \\
\hline Sin educación & 0.011 & 0.006 & 0.010 \\
\hline Primaria & 0.145 & 0.16 & 0.156 \\
\hline Secundaria & 0.49 & 0.53 & 0.519 \\
\hline Superior & 0.355 & 0.303 & 0.315 \\
\hline \multicolumn{4}{|l|}{ Zona actual } \\
\hline Urbana & 0.822 & 0.753 & 0.799 \\
\hline Rural & 0.178 & 0.247 & 0.201 \\
\hline \multicolumn{4}{|l|}{ Estrato } \\
\hline 1 & 0.376 & 0.505 & 0.420 \\
\hline 2 & 0.396 & 0.353 & 0.381 \\
\hline 3 o más & 0.228 & 0.142 & 0.199 \\
\hline \multicolumn{4}{|l|}{ ¿Tiene ingresos? } \\
\hline No & 0.401 & 0.479 & 0.427 \\
\hline $\mathrm{Si}$ & 0.599 & 0.521 & 0.573 \\
\hline $\mathrm{N}$ & 4555 & 2321 & 6876 \\
\hline
\end{tabular}

Nota: Desviaciones estándar en paréntesis. Fuente: Cálculos propios a partir de ENDS 2015 (Profamilia, 2016). 
Con el propósito de caracterizar las migrantes y su fecundidad, en la Tabla 1 se presenta un perfil de las mujeres colombianas con hijos y sin hijos por condición migratoria, considerando factores asociados a la fecundidad como la edad promedio, las razones para migrar, la zona y el estrato en los lugares de destino.

En primer lugar, se encuentra que en promedio las migrantes se concentran principalmente en las edades más fértiles (27,2 años fue el promedio de edad de las migrantes), sobre todo las migrantes con hijos (la edad promedio de las migrantes con hijos fue 26.17, mientras que la correspondiente a migrantes sin hijos fue 27.92). Esto pone de manifiesto las oportunidades de aumentar la fecundidad y de rejuvenecer composición etaria de la población económicamente activa en el lugar de destino (León, 2005).

No obstante, la movilidad de mujeres en edad joven y productiva se asocia con una redistribución territorial de la población (Ruiz, 2011). Por lo que, la pérdida de mujeres en edad productiva puede tener efectos en la reducción de mano de obra y el descenso del ritmo de reposición poblacional en los lugares de origen (Rodríguez et al., 2013).

Con respecto a las razones que motivaron la decisión de migrar, las razones familiares pesaron para aproximadamente una de cada dos migrantes mujeres (48.4\%). En específico, tuvieron una participación 8.2 puntos porcentuales más alta las mujeres migrantes con hijos, comparadas con las migrantes sin hijos (53.8\% migrantes con hijos y 45.6\% mujeres sin hijos). Por lo general, para las migrantes la reunificación familiar y el bienestar colectivo son las principales motivaciones para cambiar de lugar de residencia (Ciurlo, 2015).

Otra razón prevalente es la búsqueda de mejores ingresos, ya que fue la principal causa de migración que adujeron aproximadamente una de cada tres mujeres en la muestra (30.6\%). Seguidamente, la educación es un motivo para migrar (9.7\%), especialmente cuando existen limitaciones para educarse en el lugar de origen. Esta causa migratoria es cuatro veces mayor para las migrantes sin hijos que para las migrantes con hijos (13\% de las migrantes sin hijos, frente al 3.6\% de las migrantes con hijos); lo anterior, podría asociarse a que al no tener la responsabilidad de crianza y cuidado, pueden dedicarse a incrementar su capital humano (Fanta, Quiroga y Abeldaño, 2019).

En cuanto al estado civil, un poco más de una de cada dos migrantes se encontraban en unión conyugal (54.9\%). Así, el mayor porcentaje de las migrantes con hijos tenían pareja (75.8\% de las migrantes con hijos), mientras que el mayor porcentaje de las migrantes sin hijos no se encontraban en unión conyugal (55.9\% de las migrantes sin hijos).

Lo anterior se asocia con el ideal de reunificación familiar al migrar, pero también con aspectos culturales que proponen formar una familia con hijos y compartir la responsabilidad de la crianza como uno de los principales propósitos de unirse en pareja. Así, las migrantes con pareja tienen una mayor posibilidad de constituir núcleos familiares completos (López, 2011). Adicionalmente, el creciente reconocimiento de la independencia de la mujer para definir sus preferencias de fecundidad, la inclinación por las familias pequeñas se ha convertido en una tendencia social generalizada, 
donde aspectos como la calidad de vida y el tamaño ideal de una familia se asocian con un menor número de hijos (Paz-Gómez, 2010).

Por otra parte, el nivel educativo de las mujeres migrantes fue prevalentemente mayor que el de las nativas $(31.5 \%$ de las mujeres migrantes alcanzaron el nivel de superior, mientras que $28.5 \%$ de las mujeres nativas alcanzaron el nivel superior). En específico, entre las migrantes con hijos, la mayor proporción se presenta en el nivel educativo secundaria con una participación del 53\%, mientras que entre las migrantes sin hijos la participación en el nivel educativo secundaria alcanzó 49\%. Esto representa una desventaja para las migrantes con hijos, ya que las más escolarizadas pueden vincularse al mercado laboral con mayor eficacia (Fanta et al., 2019).

Igualmente, se destacan diferencias en la preferencia de la fecundidad entre las mujeres con nivel de educación superior de las preferencias reproductivas. Entre las mujeres migrantes con educación superior, el 35.5\% no tuvieron hijos, mientras que el 30.3\% tuvieron hijos. Esto podría estar relacionado con el hecho de que las mujeres más escolarizadas le dan una mayor importancia a la educación en su núcleo familiar, al tiempo que consideran los costos de manutención y de oportunidad que implica la maternidad (Castro y Juarez, 1995). Igualmente, niveles educativos más altos se relacionan con un mayor conocimiento acerca de métodos de planificación familiar (Paz-Gómez, 2010).

Por último, las variables asociadas al contexto socioeconómico como la zona de residencia, el estrato y si percibieron ingresos o no, permiten evidenciar los condicionantes de los hogares de las mujeres migrantes internas. De la misma forma, estas vulnerabilidades reflejan las dificultades en la adaptación de las mujeres migrantes, quienes en su mayoría tuvieron limitaciones en la inserción laboral, caracterizada por la ausencia de fuente de ingresos fijos, el desempleo, trabajos informales y salarios bajos (Ordóñez-Cuenca, 2016).

En particular, la mayor parte de las migrantes se ubicaron en zonas urbanas (79.9\%). Sin embargo, una de cada cinco migrantes con hijos se ubicaron en zonas rurales, siendo 6.9 puntos porcentuales más alta la proporción que las migrantes sin hijos (24.7\% con hijos frente al 17.8\% de las mujeres migrantes sin hijos). En cuanto al estrato, la mayor proporción de las migrantes internas (80.1\%) pertenecieron a los estratos 1 y 2 . El mayor porcentaje de las mujeres migrantes internas con hijos estaban en estrato 1 (50.5\%), mientras que el mayor porcentaje de las mujeres sin hijos se ubicaron en estrato $2(39.6 \%)$ y tuvieron una mayor proporción de migrantes en estrato 3 o superior (22.8\%\%). Igualmente, se muestra que un mayor porcentaje de mujeres sin hijos percibieron ingresos (59.9\%) en comparación con las mujeres con hijos (52.1\%).

A continuación, tras estimar el modelo logístico descrito en el apartado metodológico se procedió a estimar el efecto marginal de cada factor en la probabilidad de que una mujer migrante colombiana tuviera un hijo, con respecto al perfil de la mujer base del análisis descrita al final de la sección metodológica. En la Tabla 2 se presentan los efectos marginales estimados. 
TABLA 2.

\section{Efectos marginales del modelo logístico de la probabilidad} que una mujer migrante tenga o no hijos

\begin{tabular}{|c|c|c|}
\hline & \multicolumn{2}{|c|}{ Coeficiente } \\
\hline \multicolumn{3}{|l|}{ Tipo de migrante (ref. interno) } \\
\hline \multirow[t]{2}{*}{ Internacional } & 0.034 & \\
\hline & $(0.029)$ & \\
\hline \multirow[t]{2}{*}{ Edad } & 0.001 & \\
\hline & $(0.001)$ & \\
\hline \multicolumn{3}{|c|}{ Nivel educativo (ref. No educación) } \\
\hline \multirow[t]{2}{*}{ Primaria } & 0.08 & \\
\hline & $(0.083)$ & \\
\hline \multirow[t]{2}{*}{ Secundaria } & 0.051 & \\
\hline & $(0.082)$ & \\
\hline \multirow[t]{2}{*}{ Superior } & 0.009 & \\
\hline & $(0.083)$ & \\
\hline \multicolumn{3}{|c|}{ Razones migración (ref. Conflicto armado) } \\
\hline \multirow[t]{2}{*}{ Razones familiares } & 0.015 & \\
\hline & $(0.029)$ & \\
\hline \multirow[t]{2}{*}{ Desastres naturales } & -0.247 & $* *$ \\
\hline & $(0.114)$ & \\
\hline \multirow[t]{2}{*}{ Buscando mejorar ingresos } & -0.023 & \\
\hline & $(0.03)$ & \\
\hline \multirow[t]{2}{*}{ Educación } & -0.16 & $* * *$ \\
\hline & $(0.039)$ & \\
\hline \multirow[t]{2}{*}{ Salud } & 0.123 & $* * *$ \\
\hline & $(0.046)$ & \\
\hline \multirow[t]{2}{*}{ Otros } & 0.005 & \\
\hline & $(0.051)$ & \\
\hline \multicolumn{3}{|c|}{ Con quién viajo (ref. Acompañada) } \\
\hline \multirow[t]{2}{*}{ Sola } & -0.148 & $* * *$ \\
\hline & $(0.016)$ & \\
\hline \multicolumn{3}{|c|}{ ¿Vive en pareja? (ref. Vive en pareja) } \\
\hline \multirow[t]{2}{*}{ No vive en pareja } & -0.218 & $* * * *$ \\
\hline & $(0.02)$ & \\
\hline \multicolumn{3}{|l|}{ Zona actual (ref. Zona Urbana) } \\
\hline \multirow[t]{2}{*}{ Rural } & 0.025 & \\
\hline & $(0.017)$ & \\
\hline \multicolumn{3}{|l|}{ Estrato (ref. Estrato 1) } \\
\hline \multirow[t]{2}{*}{2} & -0.072 & $* * * *$ \\
\hline & $(0.015)$ & \\
\hline \multirow[t]{2}{*}{3 o más } & -0.133 & $* * *$ \\
\hline & $(0.021)$ & \\
\hline \multicolumn{3}{|l|}{ Ingreso (ref. No tiene) } \\
\hline \multirow[t]{2}{*}{ Sí } & -0.018 & \\
\hline & $(0.01)$ & \\
\hline
\end{tabular}

" Significativo al 10\%, ${ }^{* *}$ al 5\%, ${ }^{* * * *}$ al 1\%. Desviaciones estándar en paréntesis.

Fuente: Cálculos propios a partir de ENDS 2015 (Profamilia, 2016). 
En primer lugar, los efectos marginales de los tipos de migrantes y la edad no resultaron ser estadísticamente significativos, se realiza el análisis en los puntos porcentuales que las mujeres migrantes variaron la probabilidad de tener un hijo. De esta forma, se evidencia que las mujeres migrantes internas frente a las migrantes internacionales, tuvieron menor probabilidad de tener un hijo (3.4 puntos porcentuales más las migrantes internacionales comparadas con las migrantes internas). Por ende, la migración interna más que la migración internacional influye en las decisiones reproductivas de las mujeres, ya que disminuye su probabilidad de tener al menos un hijo.

Y esto relaciona con que, aun cuando la migración interna puede contribuir con un aumento de la fecundidad en los lugares de destino, generalmente las migrantes internacionales se mueven por razones similares a las de las migrantes internas, pero podrían tratarse de mujeres con un perfil socialmente menos vulnerable y encontrar un contexto más favorable en los países de destino (Rodríguez, 2017).

De acuerdo con la edad de las migrantes, se observa que, al aumentar la edad promedio de la mujer migrante en un año, aumenta la probabilidad de encontrar en la muestra una mujer con al menos un hijo. Sin embargo, a mayor edad se va reduciendo la probabilidad de tener un hijo. Esto se infiere con la significancia de la edad al cuadrado, que refleja la no linealidad de la relación entre edad y la probabilidad de tener un hijo. Lo anterior se relaciona con la mayor probabilidad de tener un hijo en el periodo fértil, debido al mayor riesgo que implica el embarazo en edades más avanzadas (Dressler, 2008). Además, se encuentra que, si la causa de la migración fue diferente al desplazamiento forzado por el conflicto armado se disminuye la probabilidad de tener al menos un hijo, cuando se les compara con las migrantes por desastres naturales (24.7 puntos porcentuales menos) y la educación (16 puntos porcentuales menos. Sin embargo, baja la probabilidad de las mujeres migrantes de tener un hijo, cuando la razón del traslado es por cuestiones de salud (12.3 puntos porcentuales más).

Los desafíos del proceso migratorio, antes y después del viaje, dan cuenta de la alta incidencia de los condicionantes sociales en las decisiones reproductivas de las mujeres. De esta forma, el evento migratorio surge como evento que promueve la formación y reagrupación familiar (Devolder y Bueno, 2011). Al considerar el efecto que tuvo si la migrante iba o no acompañada en su periplo migratorio en la probabilidad de tener al menos un hijo, se estima que las mujeres migrantes que viajaron solas tuvieron menor probabilidad de tener un hijo, en comparación con las que decidieron viajar acompañadas (14.8 puntos porcentuales menos). Además, para las mujeres migrantes que no vivían en unión conyugal, disminuyó en 21.8 puntos porcentuales la probabilidad de tener al menos un hijo, en comparación con las que vivían con su pareja.

Finalmente, en la medida en la que las mujeres migrantes pertenecieron a un estrato socioeconómico más alto, disminuye la probabilidad de encontrar en la muestra una mujer con al menos un hijo. De esta forma, comparadas con las del estrato 1 fue menor la probabilidad de tener un hijo en 7.2 las de estrato 2 y 13.3 puntos 
porcentuales menos las de estrato 3 o más. De esta manera, la conyugalidad aumenta el riesgo relativo de tener un hijo. Igualmente, los condicionantes socioeconómicos de la mujer, los cuales a su vez se relacionan con una mayor probabilidad de tener un hijo (Grande y del Rey, 2017). De esta forma, los condicionantes sociales en los lugares de destino podrían estar amenazando el reemplazo poblacional a lo largo del territorio colombiano.

\section{Conclusiones}

En el artículo se analiza la fecundidad de las migrantes colombianas. En específico, cómo estos dos eventos, la migración y la fecundidad, afectan el crecimiento poblacional y las disparidades demográficas y sociales a lo largo del territorio. El perfil de las mujeres migrantes internas colombianas comparadas con las migrantes internacionales, indica que pertenecieron a los estratos más bajos, una mayor proporción no percibieron ingresos, migraron principalmente en las edades más fértiles, en pareja y por razones familiares.

De esta forma, la migración interna fue menos selectiva que la migración internacional, en términos de las condiciones sociales más desfavorables. En este sentido, las mujeres migrantes internas presentaron una menor fecundidad que las mujeres migrantes internacionales, que puede estar asociado a las desventajas en el contexto social que afrontaron en los lugares de destino.

Adicionalmente, en cuanto a los factores que aumentaron la posibilidad de que las migrantes colombianas tuvieran un hijo(a) se identificaron los relacionados con las características individuales, como si se encontraban en las edades más fértiles, si alcanzaron niveles educativos más altos o si se encontraban en una unión conyugal.

De otro lado, el contexto en el cual ocurre la migración, que son estructurales y hacia los cuales deberían enfocarse las políticas públicas, se refieren a las condiciones forzadas de la migración por el conflicto armado interno, las disparidades en la calidad de vida, que en este caso se refiere a la búsqueda de acceso a salud, educación y mejores ingresos. Estas diferencias estructurales a lo largo del territorio colombiano reproducen las desigualdades que inciden en la reducción de la probabilidad de tener hijos, lo que a su vez generan cambios en la transición demográfica de los lugares de origen y de destino.

Esta investigación da cuenta de que los condicionantes de los contextos sociales a lo largo del territorio colombiano, limitan las oportunidades de las mujeres migrantes internas en los lugares de destino. En primera medida, el bienestar familiar y la falta de oportunidades de generar ingresos inhiben las posibilidades de tener hijos. La revictimización de las mujeres desplazadas forzadas por el conflicto armado interno, dado que tras su llegada aumentan las vulnerabilidades sociales, debido a las dificultades para acceder a servicios educativos, a salud sexual y reproductiva, al mercado laboral, y en general, al restablecimiento de sus derechos fundamentales tras haber sido víctimas de la violencia. 
De esta manera, es conveniente que las políticas migratorias internas vayan dirigidas a apoyar el proceso de adaptación, integración y participación de las migrantes, eliminando progresivamente las brechas que las ponen en desventaja al llegar al lugar de destino, que en gran parte son el resultado de las condiciones desfavorables en las cuales ocurre el evento migratorio.

Una limitación de esta investigación fue la imposibilidad de disponer de fuentes de información más actualizadas. Por tanto, futuras investigaciones podrían elaborar un perfil de los migrantes que enmarque las condiciones de los nuevos flujos migratorios internos e internacionales. Además, se considera relevante realizar un análisis cualitativo de lo que el evento migratorio y la fecundidad significó para las mujeres en su trayectoria de vida.

En todo caso, esta investigación muestra que se requiere mayor inversión social a lo largo de todo el territorio para equiparar y mejorar las oportunidades sociales de todos los colombianos. De esta forma, la migración y la fecundidad no seguirán causando desbalances demográficos que pueden tener repercusiones profundas en el bienestar social y en el crecimiento demográfico.

\section{Agradecimientos}

Artículo de investigación producto del proyecto de iniciación científica PIC ECO-2873, derivado del proyecto de investigación INV ECO-2968, financiados por la Vicerrectoría de Investigaciones de la Universidad Militar Nueva Granada, Vigencia 2019. Agradecemos los comentarios y contribuciones de Maite Michell Gómez Gómez, asistente de investigación del Grupo de Estudios sobre Desigualdades Sociales GeSdEs. Asimismo, agradecemos los comentarios de los revisores anónimos que contribuyeron a mejorar el artículo.

\section{REFERENCIAS}

Abu, N. (2007). Las migraciones internacionales. 'llu. Revista de ciencias de las religiones, 21, 33-50. https://doi.org/10.5209/rev_ILUR.2007.26990

Acuña, N. A. y Silva, M. O. (2016). Familia y sociedad. En, N. Aylwin y M. Solar, Trabajo social familiar (pp. 11-48). Santiago de Chile: Ediciones Universidad Católica de Chile.

Alonso, J. A. (2011). Migración internacional y desarrollo: una revisión a la luz de la crisis. Comité para Políticas de Desarrollo (CDP), 11, 1-68. Recuperado de https:/www.un.org/development/desa/dpad/wp-content/uploads/sites/45/publication/CDP-bp-2011-11-S.pdf

Bejarano, A., Guerrero, L. y Silva, C. (septiembre, 2019). Caracterización del patrón de fecundidad de las mujeres migrantes internas colombianas. Trabajo presentado al 6to Simposio Internacional de Investigación en Ciencias Económicas, Administrativas y Contables, Sociedad y Desarrollo. Universidad Libre, Bogotá, D.C., Colombia. Recuperado de http://www.unilibre.edu.co/bogota/pdfs/2019/6tosimposio/ ponencias-semilleros/23s.pdf 
Cabella, W. y Nathan, M. (2018). Los desafíos de la baja fecundidad en América Latina y el Caribe. Fondo de Población de las Naciones Unidas (UNFPA), 34. Recuperado de https://lac.unfpa.org/sites/default/files/pub-pdf/Baja fecundidad en ALC - version web español.pdf

Canales, A. (1999). Migración y urbanización en la frontera de México. En, G. Estrela y A. Canales, Ciudades de la frontera norte: migración y fecundidad (pp. 35-80). Mexicali: Universidad Autónoma de Baja California.

Castro, E. (2016). Configuración de la migración interna en la región del Paisaje Cultural Cafetero de Colombia. Revista Latinoamericana de Ciencias Sociales, Niñez y Juventud, 14(2), 1563-1585. https://doi.org/10.11600/1692715x.14246080815

Castro, T. y Juarez, F. (1995). La influencia de la educación de la mujer sobre la fecundidad en América Latina: en busca de explicaciones. Perspectivas Internacionales en Planificación Familiar, (Número especial), 4-10.

Chackiel, J. (2004). La transición de la fecundidad en América Latina 1950-2000. Papeles de Población, 10(41), 9-58. Recuperado de https://repositorio.cepal.org/ bitstream/handle/11362/12946/NotaPobla55.pdf?sequence=1

Ciurlo, A. (2015). La migración femenina y los cambios en las relaciones de género en las familias: el caso de las transmigrantes colombianas en Italia. Oasis, 21, 55-79. https://doi.org/10.18601/16577558.n21.04

CEPAL. (2006). Intersecciones entre los procesos migratorios, los derechos humanos y el desarrollo. En, Migración internacional, derechos humanos y desarrollo (pp. 17-38). Santiago de Chile: CEPAL. Recuperado de https://repositorio.cepal.org/ bitstream/handle/11362/4206/1/S2006047_es.pdf

Corona, R. (2000). Medición de la migración interestatal. Demos, (13), 8-10. Recuperado de http://revistas.unam.mx/index.php/dms/article/view/6749/6269

Devolder, D. y Bueno, X. (2011). Interacciones entre fecundidad y migración. Un estudio de las personas nacidas en el extranjero y residentes en Cataluña en 2007. Documents d'Anàlisi Geogràfica, 57(3), 441-467. https://doi.org/10.5565/ rev/dag. 244

Dressler, W. (2008). La edad de la mujer como factor de riesgo de mostalidad materna, fetal, neonatal e infantil. Revista Médica de Chile, 16(4), 457-466. https:// doi.org/10.1017/S1062798708000331

Fanta, J., Quiroga, D. E. y Abeldaño, R. (2019). La fecundidad de las migrantes del Estado Plurinacional de Bolivia, el Paraguay y el Perú en el Área Metropolitana de Buenos Aires en la primera década del siglo XXI. Notas de Población, 45(106), 91-115. https://doi.org/10.18356/51315113-es

González, J. (2012). La migración en condiciones de vulnerabilidad. En, I. Álvarez (Comp.), Mirando al norte: algunas tendencias de la migración latinoamericana (1a edición, pp. 67-76). San José: FLACSO. Recuperado de https://www.flacso. org/secretaria-general/mirando-al-norte-algunas-tendencias-migraci-n-latinoamericana 
Grande, R. y del Rey, A. (2017). La fecundidad de las mujeres latinoamericanas y caribeñas en España: ¿adaptación, mantenimiento o interrupción? Papeles de Poblacion, 23(92), 39-64. https://doi.org/10.22185/24487147.2017.92.013

Gujarati, D. N. y Porter, D. C. (2010). Modelo de regresión de respuesta cualitativa. En, Econometría (5 ed., pp. 541-589). México, D.F.: McGraw-Hill.

Ibáñez, A. M. y Velásquez, A. (2008). Impacto del desplazamiento forzoso en el bienestar de los hogares desplazados. En, A. Ibañez y A. Velásquez, El impacto del desplazamiento forzoso en Colombia: condiciones socioeconómicas de la población desplazada, vinculación a los mercados laborales y políticas públicas $\left(\mathrm{N}^{\circ}\right.$ 145, pp. 17-26). Santiago de Chile: CEPAL. Recuperado de https://repositorio. cepal.org/handle/11362/6151

Iriso, P. y Reher, D. (1987). La fecundidad y sus determinanates en España, 18871920. Un ensayo de interpretación. Revista Española de Investigaciones Sociológicas, 39, 45-118. https://doi.org/10.2307/40183293

Lagomarsino, F. (2005). ¿Cuál es la relación entre familia y migración? El caso de las familias de emigrantes ecuatorianos en Génova. En, G. Herrera, M. C. Carrillo y A. Torres (Ed.), La migración ecuatoriana. Transnacionalimo, redes e identidades (pp. 335-357). Quito: FLACSO. Recuperado de https://biblio.flacsoandes. edu.ec/catalog/resGet.php?resId=20298

León, B. (2005). La contribución demográfica de la inmigración: el caso de España. Política y cultura, (23), 121-143. Disponible en https://polcul.xoc.uam.mx/index. $\mathrm{php} / \mathrm{polcul} / \mathrm{article/view/964}$

López, L. M. (2011). Cuidado de los hijos e hijas dependientes durante la migración internacional paterna o materna. Eje cafetero, Colombia. Revista de Sociología y Antropología Virajes, (13), 257-277. Recuperado de http://vip.ucaldas.edu.co/ virajes/downloads/Virajes13_11.pdf

Mesa, C. A. y Junca, G. A. (2011). Análisis de reducción de la fecundidad: modelo de determinantes próximos. Cuadernos de Economía, 30(54), 127-150. Recuperado de https://revistas.unal.edu.co/index.php/ceconomia/article/view/24109

OIM. (2018). Informe sobre las migraciones en el mundo 2018. [Online]. Recuperado de https://publications.iom.int/system/files/pdf/wmr_2018_sp.pdf

Olea, H. (2011). Derechos Humanos y Migraciones. Un nuevo lente para un viejo fenómeno. Anuario de Derechos Humanos, 3, 197-210. https://doi.org/10.5354/07182058.2007.13482

ONU. (2017). The need for handbook on statistics of internacional migration. En, Handbook on Measuring International Migration through Population Censuses (pp. 1-15). New York: ONU. Available: https:/unstats.un.org/unsd/statcom/48thsession/documents/BG-4a-Migration-Handbook-E.pdf

Ordóñez-Cuenca, J. (2016). Tres ensayos sobre Migración y Mercado Laboral en Ecuador. [Tesis Doctoral]. Universidad de Barcelona, Barcelona, España. Disponible en http://www.tdx.cat/handle/10803/386573 
Palacios, M. (2010). La migración y los nuevos retos para el Estado-nación: relativismo ético y de identidad. Desafíos, (16), 233-254. Disponible en https://revistas. urosario.edu.co/index.php/desafios/article/view/628

Paz-Gómez, L. (2010). Tamaño de familia deseado: un análisis sobre los ideales de fecundidad en Colombia y México. Papeles de Poblacion, 16(65), 105-130. Recuperado de http://www.scielo.org.mx/pdf/pp/v16n65/v16n65a4.pdf

Perilla, C. (2011). Migración y desarrollo humano en Colombia. En, E. Hung (Ed.), Migración, desarrollo humano e internacionalización (pp. 7-19). Barranquilla: Editorial Universidad del Norte. Recuperado de https://www.uninorte.edu.co/ documents/72553/6665473a-4eb7-47eb-965a-69ab344d3d33

Profamilia. (2016). Migración colombiana durante los últimos años. Encuesta Nacional de Demografía y Salud, ENDS 2015, 5-6. Recuperado de https://www. minsalud.gov.co/Documents/General/20161212_B_ENDS 2015_Migracioon.pdf

Ramírez, C., Zuluaga, M. y Perilla, C. (2010). Perfil Migratorio de Colombia. En Organización Internacional para las Migraciones - OIM Colombia. Bogotá, D.C.: OIM. Recuperado de https://robuenosaires.iom.int/sites/default/files/publicaciones/Perfil-Migratorio-de-Colombia.pdf

República de Colombia. Ministerio de Salud y Protección Social y Profamilia. (2015). Componente demográfico. En Encuesta Nacional de Demografía y Salud. (Tomo I, pp. 37-44). Bogotá, D.C.: Minsalud. Recuperado de https://profamilia.org.co/ wp-content/uploads/2018/12/ENDS-TOMO-I.pdf

Rodríguez, D. (2017). Un marco teórico para el estudio de la fecundidad de las migrantes cubanas en Estados Unidos. Revista Novedades en Población, 13(26), 10-23. Disponible en http://www.novpob.uh.cu/index.php/NovPob/article/view/261

Rodríguez, J. (2015). Migración interna de la población joven: el caso de América Latina. Revista Latinoamericana de Población, 2(3), 9-26. https://oi.org/10.31406/ relap2008.v2.i2.n3.4

Rodríguez, J. (abril, 2007). Migración interna y desarrollo: el caso de América Latina. Presentado en el taller Migración interna y desarrollo en Brasil: diagnóstico, perspectivas y políticas. CEPAL, Brasilia, Brasil. Recuperado de https:// www.cepal.org/sites/default/files/courses/files/jrodriguez_1.pdf

Rodríguez, J. (2004). Enfoques y modelos conceptuales sobre la migración interna. En, J. Rodríguez, Serie Población y Desarrollo. Migración interna en América Latina y el Caribe: estudio regional del periodo 1980-2000 (50, pp. 19-32). Santiago de Chile: CEPAL. Recuperado de https://repositorio.cepal.org/bitstream/ handle/11362/7188/1/S04117_es.pdf

Rodríguez, D., Turra, C., Bonifácio, G. y Wajnman, S. (2013). Efectos de la migración sobre el crecimiento poblacional a largo plazo de las provincias cubanas. Revista Brasileira de Estudos de Populacao, 30(2), 429-444. https://doi.org/10.1590/ S0102-30982013000200005 
Rodríguez, J. (2008). Distribución espacial, migración interna y desarrollo en América Latina y el Caribe. Revista de la CEPAL, 96, 135-155. https://doi.org/10.18356/ cd7c0ed5-es

Romero, J. E. (2015). El éxito económico de los costeños en Bogotá: migración interna y capital humano. Documentos de Trabajo sobre Economía Regional y Urbana. Población y desarrollo en el pacífico colombiano, (232), 255-283. https://doi. org/10.32468/dtseru.129

Ruiz, N. (2011). El desplazamiento Forzado en Colombia: una revisión histórica y demográfica. Estudios Demograficos y Urbanos, 26(1), 141-177. https://doi. org/10.24201/edu.v26i1.1400

Schkolnik, S. y Chackiel, J. (2004). Los sectores rezagados en la transición de la fecundidad en América Latina. Revista de la CEPAL, (83), 13-31. https://doi. org/10.18356/50d6fc1c-es

Silva, A. C. y González, P. (2007). Una revisión a la composición y transición demográfica en Colombia. Revista Facultad de Ciencias Económicas: Investigación y Reflexión, 15(2), 61-78. Disponible en https://revistas.unimilitar.edu.co/index. $\mathrm{php} / \mathrm{rfce} / \mathrm{article/view/4533}$

Silva, A. C. y Sarmiento, J. A. (2013). Desplazados forzados y su participación en el mercado laboral colombiano. Revista Facultad de Ciencias Económicas, 21(1), 167-187. https://doi.org/10.18359/rfce.672

UNFPA. (2006). Desigualdades y derechos. En, H. González (Ed.), Población, desigualdad y políticas públicas: un diálogo político estratégico (pp. 55-94). Caracas: CDB Publicaciones.

Vicente, T. L. (2006). Importancia de los flujos migratorios de mujeres. En, C. Blanco (Coord.), Migraciones: nuevas movilidades en un mundo en movimiento (pp. 206-233). Barcelona: Anthropos.

Willers, S. (2016). Migración y violencia: las experiencias de mujeres migrantes centroamericanas en tránsito por México. Sociológica (México), 31(89), 163-195. Disponible en http:/www.sociologicamexico.azc.uam.mx/index.php/Sociologica/ article/view/1295

Laura Alejandra Guerrero Cely es Economista de la Universidad Militar Nueva Granada (Colombia). Participante activo durante el periodo 2017-2019 del Semillero de estudios Macroeconómicos perteneciente al Grupo de Investigación GESDES de la Universidad Militar Nueva Granada. Auxiliar de Investigación en el Grupo de Investigación GESDES, de la Universidad Militar Nueva Granada, desde abril del 2019 hasta febrero del 2020. https://orcid.org/0000-0003-2480-6458

Alisson Zharick Bejarano Carreño es Economista de la Universidad Militar Nueva Granada (Colombia). Participante activo durante el periodo 2017-2019 del Semillero de estudios Macroeconómicos perteneciente al Grupo de Investigación GESDES de la Universidad Militar Nueva Granada. https://orcid.org/0000-0001-8348-7878 
Adriana Carolina Silvia Arias es Economista de la Universidad del Rosario (Colombia), Maestra en Economía de la Universidad de los Andes (Colombia) y Doctora en Estudios de Población de El Colegio de México. Profesora-investigadora de la Facultad de Ciencias Económicas de la Universidad Militar Nueva Granada. Miembro de la Red Trabajo y condiciones laborales - Tetra y de la Red Población y Trabajo de la Asociación Latinoamericana de Población. https://orcid.org/0000-0003-1610-881X 